\title{
Dynamical role of Polyakov loops in the QCD thermodynamics
}

\author{
N.O. Agasian ${ }^{a, b, *}$, M.S. Lukashov ${ }^{a, c, \dagger}$ and Yu.A. Simonov ${ }^{a, \ddagger}$ \\ a Alikhanov Institute for Theoretical and Experimental Physics, \\ Moscow 117218, Russia \\ b National Research Nuclear University "MEPhI", \\ Moscow 115409, Russia \\ ${ }^{c}$ Moscow Institute of Physics and Technology, \\ Dolgoprudny 141700, Moscow Region, Russia
}

July 9,2021

\begin{abstract}
Polyakov loops $L_{a}(T), a=3,8, \ldots$ are shown to give the most important nonperturbative contribution to the thermodynamic potentials. Derived from the gluonic field correlators they enter as factors into free energy. It is shown in the $S U(3)$ case that $L_{a}(T)$ define to a large extent the behavior of the free energy and the trace anomaly $I(T)$, most sensitive to nonperturbative effects.
\end{abstract}

Keywords: phase transition; Polyakov line; trace anomaly

PACS numbers: 11.15.Tk, 12.38.Lg

*agasian@itep.ru

†lukashov@phystech.edu

†simonov@itep.ru 
1. Polyakov lines (PL) $L_{a}(T), a=3,8, \ldots$ play a double role in the dynamics of hot QCD. First of all, they serve as an order parameter (see [1,2] for reviews) being nonzero above the critical temperature and signalling the absence of confinement (e.g. for the adjoint PL in the $\mathrm{SU}(3)$ theory, there is a strong jump in the values of PL at $T=T_{c}[3]$ ). Secondly, as we stress below, PL have an important role in the whole dynamics of the hot QCD. In the Field Correlator (FC) approach this was directly derived from the basic QCD Lagrangian with account of the quadratic gluon field correlators [4,5]. It was shown in [4,5] that the free energy is proportional to the $L^{n}$ in the Matsubaru series over $n$. As will be shown below, this dependence is crucial in defining behavior of all thermodynamic quantities in the region $T_{c} \leq T \lesssim 4 T_{c}$ and is substantial for $T \lesssim 10 T_{c}$. In particular, the remarkable plateau of $\frac{I(T)}{T^{2} T_{c}^{2}}$ in $\mathrm{SU}(3)$, discovered in [6], is for the most part due to the $1 / T^{2}$ behavior of $T \frac{\partial}{\partial T} L_{a d j}(T)$.

In other approaches to the hot QCD dynamics the role of PL was also taken into account in different ways, e.g. in the matrix PL models [7], and in the PNJL model [8 10], introducing an additional potential $V\left(L, L^{+}\right)$in the Lagrangian, see [11] for a review.

2. The quadratic gluon field correlator consists of two colorelectric terms $D^{E}$ and $D_{1}^{E}[12$,

$$
\begin{gathered}
D_{\mu \nu \lambda \sigma}(x, y) \equiv g^{2} \operatorname{tr}_{a}\left\langle F_{\mu}(x) \Phi F_{\lambda \sigma}(y) \Phi\right\rangle= \\
=c_{a}\left\{\left(\delta_{\mu \sigma} \delta_{\nu \sigma}-\delta_{\mu \sigma} \delta_{\nu \lambda}\right) D(x-y)+\right. \\
\left.+\frac{1}{2}\left[\frac{\partial}{\partial x_{\mu}}\left(x_{\lambda} \delta_{\nu \sigma}-x_{\sigma} \delta_{\nu \lambda}\right)+(\mu \lambda \leftrightarrow \nu \sigma)\right] D_{1}(x-y)\right\},
\end{gathered}
$$

and the resulting nonperturbative (np) plus perturbative interaction between color objects in the repr. $a$ can be written as 12

$$
\begin{aligned}
& V_{a}(r)= c_{a}\left\{2 \int_{0}^{r}(r-\lambda) d \lambda \int_{0}^{\infty} d \nu D^{E}(\lambda, \nu)+\right. \\
&+\left.\int_{0}^{r} \lambda d \lambda \int_{0}^{\infty} d \nu D_{1}^{E}(\lambda, \nu)\right\}= \\
&=c_{a}\left\{V_{\text {conf }}(r)+V_{1}(r)\right\}, c_{3}=1, c_{8}=\frac{9}{4}, \text { etc. }
\end{aligned}
$$

In the deconfinement phase $\left(V_{\text {conf }}=0\right), V_{1}(r)$ has an important property that $V_{1}(\infty)=$ const, which implies, that each deconfined gluon (or quark) goes astray with a piece of energy $\frac{c_{a}}{2} V_{1}(\infty)$. It is important, that this term appears in the gluon pressure in the exponent, $\exp \left(-c_{a} \frac{V_{1}(\infty)}{2 T}\right)$ as follows from the path integral form of the gluon pressure [4]

$$
P_{g l}=\left(N_{c}^{2}-1\right) \int_{0}^{\infty} \frac{d s}{s} \sum_{n} G^{(n)}(s),
$$

where $G^{(n)}(s)$ is the winding path integral over the loop $C_{n}$, where all gauge field dependence enters as

$$
\begin{aligned}
G^{(n)}(s) & \sim\left\langle\exp \left(i g \int_{C_{n}} d z_{\mu} A_{\mu}\right)\right\rangle=\exp \left(-\frac{1}{2} \int_{S_{n}} d \sigma_{\mu \nu}(u) \times\right. \\
& \left.\times \int_{S_{n}} d \sigma_{\lambda \sigma}(u)\left\langle F_{\mu \nu} \Phi F_{\lambda \sigma} \Phi\right\rangle+O\left(F^{4}\right)\right)
\end{aligned}
$$


Insertion of the field correlator (1) in (4) produces exactly the integral

$$
\begin{aligned}
J(T, r)= & \exp \left(-\frac{c_{a}}{2} \int_{0}^{1 / T} d t_{E} V_{1}(r, T)\right)= \\
& =\exp \left(-\frac{c_{a}}{2 T} V_{1}(r, T)\right) .
\end{aligned}
$$

Following [13], it is convenient to extract from $V_{1}(r, T)$ the large distance limit $V_{1}(\infty, T)$, leaving the sum of the attractive interactions $\Delta V_{1}=V_{1}(r, T)-V_{1}(\infty, T)$ and the renormalized perturbative interaction $V_{1}^{C}(r, T)$ to account for as a correction. As a result in the leading approximation the function $J(T, r)$ in (5) acquires a factor $J(T, \infty)$, entering in $G^{(n)}(s)$ and $P_{g l}(T)$, which we call the Polyakov line $L_{a}(T)$

$$
\begin{gathered}
L_{a}(T)=\exp \left(-\frac{c_{a}}{2} \frac{V_{1}(\infty, T)}{T}\right) \\
G^{(n)}(s)=\frac{1}{\sqrt{4 \pi s}} e^{-\frac{n^{2}}{4 s T^{2}}} G_{3}(s) L_{8}^{n}(T),
\end{gathered}
$$

and $G_{3}(s)$ is the $3 \mathrm{~d}$ path integral over the $3 \mathrm{~d}$ portion of the loop $C_{n}$

$$
G_{3}(s)=\int\left(D^{3} z\right)_{x x} e^{-K_{3 d}}\left\langle W_{3}\right\rangle
$$

Here the $3 \mathrm{~d}$ projected Wilson loop $\left\langle W_{3}\right\rangle$ obeys the spatial area law with the colormagnetic string tension $\sigma_{s}$ and the $3 \mathrm{~d}$ area $A_{3}$

$$
\left\langle W_{3}\right\rangle=\exp \left(-\sigma_{s} A_{3}\right)
$$

In [4,5] $G_{3}(s)$ was calculated in the approximation when $\sigma_{s}=0$, and as a result one has $G_{3}(s)=\frac{1}{(4 \pi s)^{3 / 2}}$, and

$$
\begin{aligned}
P_{g l}^{(0)} & =\frac{2\left(N_{c}^{2}-1\right)}{\pi^{2}} T^{4} \sum_{n=1}^{\infty} \frac{1}{n^{4}} L_{8}^{n}= \\
& =\frac{2\left(N_{c}^{2}-1\right)}{\pi^{2}} T^{4} \operatorname{Li}_{4}\left(L_{8}\right)
\end{aligned}
$$

which for $L_{8}=1$ yields the Stefan-Boltzmann result $P_{g l}^{(S B)}=\frac{\left(N_{c}^{2}-1\right) \pi T^{4}}{45}$, defining the asymptotic behavior of $P_{g l}$, when $V_{1}$ decreases at large $T$.

Note several important points in our definition of $L_{a}(T)$ :

i) $L_{a}(T)$ automatically satisfies the Casimir scaling law due to factor $c_{a}$ in (21), this scaling is supported by lattice data $[3,14]$.

ii) In the correlator $P(\mathbf{x}-\mathbf{y})$ of two Polyakov loops, studied in [13], one obtains the same form as in (4) with the loops $\left(S_{n}, S_{n}\right) \rightarrow\left(S_{n}, S_{n}^{\prime}\right)$ referring to two different loops at the distance $r=|\mathbf{x}-\mathbf{y}|$ from each other, and one obtains the same form as in [15, 16]

$$
\begin{aligned}
P(\mathbf{x}-\mathbf{y}) & =\frac{1}{N_{c}^{2}} \exp \left(-\frac{\tilde{F}_{1}(r, T)}{T}\right)+ \\
& +\frac{N_{c}^{2}-1}{N_{c}^{2}} \exp \left(-\frac{\tilde{F}_{8}(r, T)}{T}\right),
\end{aligned}
$$


where e.g. $\tilde{F}_{1}(r, T)=c_{a}\left(V_{1}(r, T)+V_{\text {conf }}(r, T)\right)$. As a result $P(r)$ vanishes in the confining phase for $r \rightarrow \infty$ and is a product of two Polyakov loops in this limit in the deconfined phase, as it should be. This exercise also implies that the Polyakov loop enters in $P_{g l}^{(0)}$, Eq. (10), in the approximation, when the interaction $V_{1}(r, T)$ between neighboring gluons is replaced by $V_{1}(\infty, T)$.

iii) The definition (6) of PL appears due to the vacuum average of gluonic field, Eq. (11), which evidently violates the $Z(3)$ symmetry.

3. As was stated above the resulting gluon pressure $P_{g l}$ in the lowest approximation is given by

$$
\begin{aligned}
P_{g l} & =\frac{\left(N_{c}^{2}-1\right)}{\sqrt{4 \pi}} \int_{0}^{\infty} \frac{d s}{s^{3 / 2}} \times \\
& \times \sum_{n=1,2, \ldots} e^{-\frac{n^{2}}{4 s T^{2}}} G_{3}(s) L_{8}^{(n)}(T),
\end{aligned}
$$

and the point is where to find the information about PL. This can be obtained from several sources:

a) From the lattice data on $D_{1}(x)$ in [17-19], where it was found that the correlator $D_{1}(x)$, unlike $D(x)$, does not vanish above $T_{c}$, and decays as $\exp (-M|x|)$ with $M=O(1 \mathrm{GeV})$.

The corresponding values of $V_{1}(R, T)$ were calculated from $D_{1}$ in the interval $1.007 \leq$ $T / T_{c} \leq 1.261$ in [20], however with low accuracy.

b) From the gluelump representation of $D_{1}(x)$ in [21] one finds in [13] that the np part of $V_{1}$ can be represented as

$$
\begin{gathered}
V_{1}^{(n p)}(\infty, T)=\frac{d}{M_{1}}\left[1-\frac{T}{M_{1}}\left(1-e^{-M_{1} / T}\right)\right], \\
d=0.432 \mathrm{GeV}^{2}, M_{1}=0.69 \mathrm{GeV} .
\end{gathered}
$$

This form agrees with lattice data [22] and can be used to define $L_{a}(T)$ at least for $T<2 T_{c}$.

c) From the free energies $F_{i}(r, T)$, obtained from the PL correlator [15, 16, 22], which have the same form as in (11) with the replacement $\tilde{F}_{i} \rightarrow F_{i}$. This replacement implies, as was stated in [13], that the lattice version of $V_{1}(r, T)$ is the singlet free energy $F_{1}(r, T)$, which is an averaged value over all excited states, yielding the inequality $F_{1}(r, T)<V_{1}(r, T)$. As a result one obtains $L_{a}(T)$ in (6), which satisfies the condition $L_{a}(T)<L_{a}^{\text {lat }}(T)$, where $L_{a}^{\text {lat }}(T)$ is found on the lattice via $F_{1}(\infty, T)$. In particular, $F_{1}(\infty, T)$ becomes negative for $T>2 T_{c}$, yielding $L_{a}^{\text {lat }}(T)>1$, while in our case for all $T L_{a}(T)<1$. In what follows we are using the form $V_{1}(T)$, which is close to that in [4,5] and the resulting $L_{a}(T)$ is close the lattice data of [3] for $T \leq 2 T_{c}$, namely

$$
V_{1}(\infty, T)=\frac{0.13 \mathrm{GeV}}{T / T_{c}-0.84} .
$$

4. In the previous section we have disregarded the colormagnetic interaction $(\mathrm{CM})$ contained in $G_{3}(s)$ in (12). To account for the CM effects, one should calculate $G_{3}(s)$ in (8), 
where $K_{3 d}=\frac{1}{4} \int_{0}^{s} \sum_{i=1}^{3}\left(\frac{d z_{i}}{d \tau}\right)^{2} d \tau$. As it is seen from (8)), what one should estimate is the gluon loop in 3d, covered with the confining film with string tension $\sigma_{s}(T)$. Using the same method as in [23, 24], one can calculate $G_{3}(s)$ in terms of the $2 \mathrm{~d}$ gluon-gluon bound states with masses $M_{\nu}=4 \omega_{\nu}^{(0)}$, where $\omega_{\nu}^{(0)}=\frac{3}{2}\left(\frac{a_{\nu}}{3}\right)^{3 / 4} \sqrt{\sigma(T)}, \nu=0,1,2, \ldots, a_{0}=1.74$ namely

$$
G_{3}(s)=\frac{1}{\sqrt{\pi s}} \sum_{\nu=0,1, . .} \varphi_{\nu}^{2}(0) e^{-M_{\nu} \omega_{\nu}^{(0)} s}
$$

where $\varphi_{\nu}(0)$ is the $2 \mathrm{~d}$ wave function at origin. From dynamical consideration $\varphi_{\nu}^{2}(0)=c_{\nu} \sigma_{s}(T)$, with $c_{\nu}$ - numerical constant. Moreover, $M_{\nu} \omega_{\nu}^{(0)} \cong 4 \sigma_{s}(T) \approx m_{D}^{2}(T)$, where $m_{D}(T)$ is the np Debye screening mass, calculated in [23,24] in agreement with lattice data [25]. Thus keeping the lowest term with $\nu=0$ in (15) one has $G_{3}^{(\min )}(s)=\frac{1}{\sqrt{\pi s}} c_{0} \sigma_{s} e^{-m_{D}^{2} s}$ and inserting this into (12) one has

$$
\begin{aligned}
P_{g l}^{(\min )}(T) & =\frac{\left(N_{c}^{2}-1\right) c_{0} \sigma_{s} m_{D} T}{2 \pi^{2}} \times \\
& \times \sum_{n=1,2, \ldots} \frac{1}{n} K_{1}\left(\frac{n m_{D}}{T}\right) L_{8}^{n} .
\end{aligned}
$$

It was shown in [26, 27] that

$$
\sqrt{\sigma_{s}(T)}=c_{\sigma} g^{2}(T) T
$$

where use was made of the two-loop expression for $g^{2}(T)$

$$
\begin{gathered}
g^{-2}(T)=2 b_{0} \ln \frac{T}{\Lambda_{\sigma}}+\frac{b_{1}}{b_{0}} \ln \left(2 \ln \frac{T}{\Lambda_{\sigma}}\right), \\
b_{0}=\frac{11 N_{c}}{48 \pi^{2}}, \quad b_{1}=\frac{34}{3}\left(\frac{N_{c}}{16 \pi^{2}}\right)^{2} .
\end{gathered}
$$

The two constants $c_{\sigma}$ and $\Lambda_{\sigma}$ were determined using a two-parameter fit to lattice results. For the $\mathrm{SU}(3)$ gauge theory $c_{\sigma}=0.566 \pm 0.013, \Lambda_{\sigma}=(0.104 \pm 0.009) T_{c}[26,27$.

At large $T, \sigma_{s}(T)$ behaves as $c_{\sigma}^{2} g^{4}(T) T^{2}$, where $g^{2}(T)$ is $O\left(\frac{1}{\ln \frac{T}{\Lambda_{\sigma}}}\right)$ (however $c_{\sigma}$ is a np quantity [28]), and as a result $P_{g l}^{(\min )}(T) / T^{4} \sim O\left(\frac{1}{\ln ^{2} \frac{T}{\Lambda_{\sigma}}}\right)$. This amounts to the approximately $50 \%$ decrease of $P_{g l}^{(\min )}$ from $T=2 T_{c}$ to $T=5 T_{c}$, therefore it is important to consider also the higher states in the sum over $\nu$.

To account for higher states it is convenient to exploit the oscillator form of the colormagnetic interaction, which immediately produces the analytic answer, namely

$$
G_{3}(s)=\frac{1}{(4 \pi s)^{3 / 2}} \frac{M_{0}^{2}}{\operatorname{sh} M_{0}^{2} s}
$$

where $M_{0}=\omega$ in the lowest excitation in the oscillator potential, which we can associate with the screening mass $m_{D}=2 \sqrt{\sigma_{s}}[23,24]$. 
Inserting (19) in (12) one obtains the final form of the gluon pressure with account of the spatial confinement in the oscillator form

$$
P_{g l}^{(o s c)}=\frac{2\left(N_{c}^{2}-1\right)}{(4 \pi)^{2}} \sum_{n=1}^{\infty} L_{8}^{n} \int \frac{d s}{s^{2}} e^{-\frac{n^{2}}{4 s T^{2}}} \frac{M_{0}^{2}}{\operatorname{sh} M_{0}^{2} s} .
$$

Note, that in the limit $M_{0}^{2} \rightarrow 0$ one recovers the free case, Eq. (10).

One can also use the oscillator form, reproducing the linear confinement with the accuracy of $5 \%$; this corresponds to the replacement in (20) $: \frac{M_{0}^{2}}{\operatorname{sh} M_{0}^{2} s} \rightarrow \frac{1}{s}\left(\frac{M_{0}^{2} s}{\operatorname{sh} M_{0}^{2} s}\right)^{1 / 2}$. This modified oscillator form we are using below in our calculations. However the final result is almost (within few percent) insensitive to this replacement.

5. The results of numerical calculation of the pressure in the approximations: $P_{g l}^{(0)}(T)$ and $P_{g l}^{(o s c)}(T)$ with $L_{8}(T)$ using (14) are given in the Fig. 1, in comparison with the lattice data of [6]. One can see an improvement of the results, when $\sigma_{s}(T)$ is taken into account in $P_{g l}^{(o s c)}(T)$, however already $P_{g l}^{(0)}(T)$, where only $L_{8}(T)$ is taken into account, is a reasonable approximation. This supports our main idea, that PL are the important dynamical input, which should enter $P_{g l}$ as factors, according to our derivation.

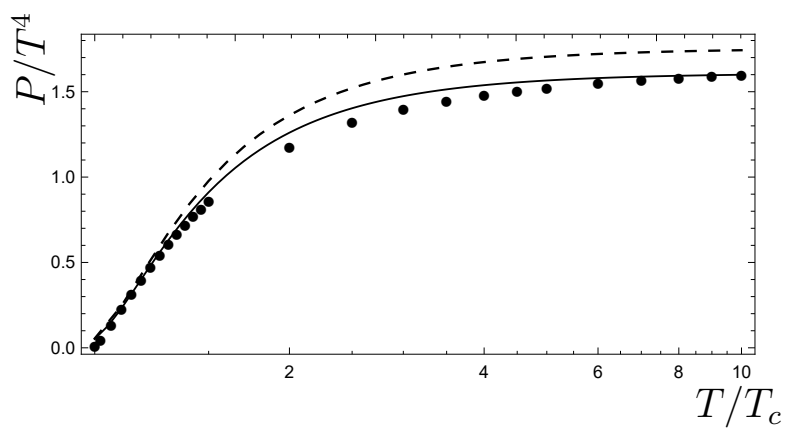

Figure 1: The pressure $\frac{P(T)}{T^{4}}$ in the $\mathrm{SU}(3)$ theory. The dashed line corresponds to the pressure without magnetic confinement Eq.(10). The solid line is for the modified oscillator confinement, and filled dots are for the lattice data [6].

Leaving details of comparison, as well as entropy $s(T)$, internal energy $\varepsilon(T)$ and sound velocity $c_{s}(T)$ to another publication [29, we shall consider in more detail the scale anomaly $I(T)=\varepsilon-3 P$, which can be written as

$$
\frac{I(T)}{T^{4}}=T \frac{\partial}{\partial T}\left(\frac{P_{g l}}{T^{4}}\right)=\frac{\bar{I}(T)}{T^{4}}+p_{g l}(T) \frac{T \partial L_{8}}{\partial T}
$$

where we write $P_{g l}$ as $\frac{P_{g l}}{T^{4}}=p_{g l} L_{8}(T)$, and

$$
\bar{I}(T)=T \frac{\partial p_{g l}}{\partial T} L_{8}(T)
$$

In Fig. 2 we show $\frac{I(T)}{T^{4}}$ and $\frac{I(T)}{T^{4}}\left(\frac{T}{T_{c}}\right)^{2}$ as functions of $T$ in the interval $T_{c} \leq T \leq 10 T_{c}$, and note, that as was found on the lattice in [6], this purely np phenomenon, discovered in [6] is well reproduced by mostly the properties of $\frac{\partial L_{8}(T)}{\partial T}$ which behaves in this region as $1 / T^{2}$. 


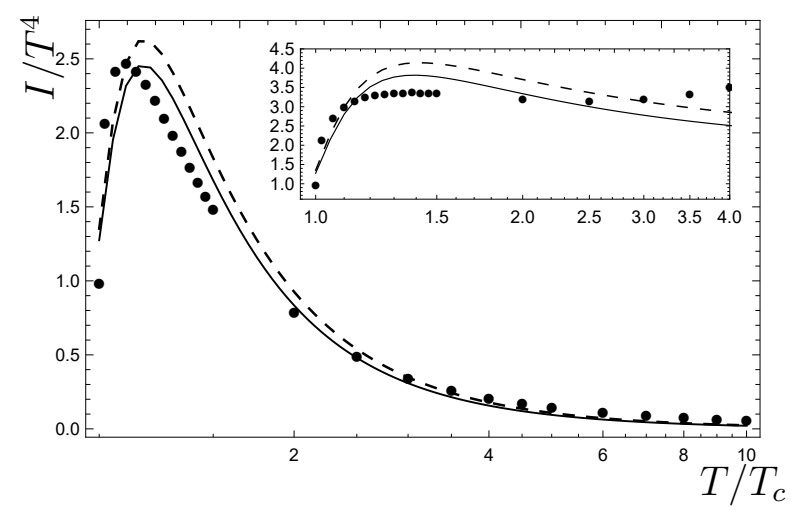

Figure 2: The trace anomaly $\frac{I(T)}{T^{4}}$. Notations are the same as in Fig.1. In the upper right corner the plot is given for $\frac{I(T)}{T^{4}}\left(\frac{T}{T_{c}}\right)^{2}$.

Our purpose in this paper was to demonstrate the dynamical importance of the Polyakov loops in the QCD thermodynamics in the SU(3) case. We have also shown in some detail that PL enter thermodynamic potentials as factors and contain a most part of np dynamics, which allows to explain the spectacular shoulder in the $\frac{I(T)}{T^{4}}\left(\frac{T}{T_{c}}\right)^{2}$ dependence.

This work was supported by a grant from the Russian Science Foundation (project number 16-12-10414).

\section{References}

[1] P. Petreczky, J. Phys. G 39, 093002 (2012).

[2] S. Sharma, Adv. High Energy Phys. 2013, 452978 (2013).

[3] S. Gupta, K. Hübner and O. Kaczmarek, Nucl. Phys. A 785, 278 (2007).

[4] Yu.A. Simonov, Ann. Phys. (NY) 323, 783 (2008).

[5] E.V. Komarov and Yu.A. Simonov, Ann. Phys. (NY) 323, 1230 (2008).

[6] S. Borsanyi, G. Endrödi, Z. Fodor, S.D. Katz and K.K. Szabo, JHEP 1207, 056 (2012).

[7] R.D. Pisarski, Phys. Rev. D 74, 121703 (2006).

[8] P.N. Meisinger, M. Ogilvie and T.B. Miller, Phys. Lett. B 585, 149 (2004).

[9] K. Fukushima, Phys. Rev. D 68, 045004 (2003).

[10] D. Blaschke, M. Buballa, A.E. Radzhabov and M.K. Volkov, Phys. Atom. Nucl. 71, 1981 (2008).

[11] J.O. Andersen, T. Brauner and W.R. Naylor, Phys. Rev. D 92, 114504 (2015).

[12] H.G. Dosch, Yu.A. Simonov, Phys. Lett. B 205, 339 (1988). 
[13] Yu.A. Simonov, Phys. Lett. B 619, 293 (2005).

[14] A. Mykkanen, M. Panero and K. Rummukainen, JHEP 1205, 069 (2012).

[15] L.D. McLerran and B. Svetitsky, Phys. Rev. D 24, 450 (1981).

[16] S. Nadkarni, Phys. Rev. D 34, 3904 (1986).

[17] M. D’Elia, A. Di Giacomo and E. Meggiolaro, Phys. Lett. B 408, 315 (1997).

[18] M. D'Elia, A. Di Giacomo and E. Meggiolaro, Phys. Rev. D 67, 114504 (2003).

[19] G.S. Bali, N. Brambilla and A. Vairo, Phys. Lett. B 421, 265 (1998).

[20] A. Di Giacomo, E. Meggiolaro, Yu.A. Simonov, A.I. Veselov, Phys. Atom. Nucl. 70, 908 (2007).

[21] Yu.A. Simonov, Nucl. Phys. B 592, 350 (2001).

[22] O. Kaczmarek, F. Karsch, P. Petreczky and F. Zantow, Phys. Lett. B 543, 41 (2002).

[23] N.O. Agasian, Yu.A. Simonov, Phys. Lett. B 639, 82 (2006).

[24] N.O. Agasian, Phys. Lett. B 562, 257 (2003).

[25] O. Kaczmarek, F. Zantow, Phys. Rev. D 71, 114510 (2005).

[26] F. Karsch, E. Laermann and M. Lütgemeier, Phys. Lett. B 346, 94 (1995).

[27] G. Boyd et al., Nucl. Phys. B 469, 419 (1996).

[28] Yu.A. Simonov, arXiv: 1605.07060.

[29] N.O. Agasian, M.S. Lukashov and Yu.A. Simonov, (in preparation). 